\title{
Spatial analysis to create a walkability index. Case study: San Juan Street, Medellín
}

\author{
Sandra Milena Salazar Martinez \\ Universidad Pontificia Bolivariana | Colombia | sandra.salazar@upb.edu.co \\ Luis Miguel Rios Betancur \\ Universidad Pontificia Bolivariana | Colombia | luismiguel.rios@upb.edu.co \\ Luis Daniel Santana Rivas \\ Universidad Nacional de Colombia | Colombia | Idsantanar@unal.edu.co
}

\begin{abstract}
To walk represents one of the main ways of commuting, either by necessity or pleasure, it is a guaranteed way for everyone and it contributes significantly to the health and well-being of people. However, Medellín's urban space design is not planned to improve walking conditions. In this context, a spatial index was built based on the components that influence the urban scene, which allows to evaluate the walking experience, not only its possibility, on an axis of the city. It concludes how these variables prioritize other actions and strategies that must be taken into consideration to build a walkable city.
\end{abstract}

Keywords: Walkability; Spatial Analysis; Geographic information systems Urban Scene; Medellín.

\section{INTRODUCCIÓN}

Medellín ha presentado en los últimos años varios eventos de emergencia ambiental por la contaminación del aire. La población ha aumentado y en mayor proporción la del parque automotor; por otro lado, la encuesta origen destino realizada para todos los municipios del Valle de Aburrá, en el 2017 evidencia que el $27 \%$ de los viajes se realiza a pie, mientras que un $31 \%$ de los viajes se realiza por medios motorizados no masivos. El $19 \%$ se realiza en transporte público y el $15 \%$ en metro y metroplús (AMVA, 2017). Estos porcentajes indican la alta dependencia a medios motorizados altamente contaminantes para los desplazamientos cotidianos. La mayor parte de las calles de la ciudad no ofrecen condiciones óptimas para que las personas puedan caminar con agrado, salud y confort, lo que facilita la exclusión de esta opción y que se privilegie el transporte vehicular en recorridos que, por su distancia, se podrían realizar caminando.

La importancia de caminar en la ciudad y de reiterar en que las ciudades deben orientar sus políticas para que esta actividad se incremente, es un tema ya común a nivel mundial. En los últimos años se han incrementado los estudios sobre las condiciones para caminar en la ciudad, y se puede encontrar una literatura abundante sobre el tema, donde se centran en su mayoría sobre las variables espaciales como continuidad, ancho del andén y las condiciones de los cruces, pero poco valoran cómo influyen las condiciones del paisaje urbano para la caminata (Ewing et al., 2016). Las condiciones para caminar en las ciudades de América Latina y específicamente Colombia, no son las más adecuadas, y en parte esto es debido al rápido crecimiento que experimentaron las ciudades. Este crecimiento estuvo orientado al espacio para el vehículo, se aumentaron los carriles vehiculares, pero no el espacio para el peatón, que sigue conservando las mismas dimensiones de cuando en la ciudad se transitaba con caballos y bicicleta. Hoy, cuando las políticas públicas buscan promover la caminata, las personas siguen optando como primera opción por sus automóviles y esto se debe en parte, a que las calles de la ciudad no los invita a caminar.

Este artículo es producto del proyecto de investigación universitario "La ciudad caminable. Estrategias de diseño y recuperación de la calle como espacio público en las ciudades de Medellín y Montería". El proyecto se ha realizado durante los años 2019 y 2020 y ha contemplado tres etapas de investigación: la revisión de literatura que ha permitido acercarse al problema de estudio y determinar las variables de trabajo para el análisis, una segunda fase de estudio del área de trabajo y experimentación, donde se exploraron distintas maneras de abordar las áreas seleccionadas que incluyeron selección de segmentos de las calles donde se realizaron algunas pruebas de trabajo de campo con estudiantes, vuelos dron sobre las áreas críticas y cómo última etapa, la construcción del índice de caminata que permitió la valoración de toda la calle y que cuyos resultados se presentan en este paper. El equipo de trabajo está constituido por tres arquitectos y un geógrafo, todos con especialidades distintas dentro del campo del urbanismo y 
análisis espacial a través de los sistemas de información cartográfica.

Dentro de los objetivos de la investigación no estaba contemplada la realización de un trabajo de campo, por lo cual se debía buscar un modelo de análisis que permitiera homogenizar criterios válidos para evaluar la caminata en corredores urbanos, a partir de datos que se pudieran obtener en diferentes ciudades, entendiendo la dificultad para acceder a la información en el contexto colombiano. Buscar una comparación con Montería, como ciudad intermedia, ponía el reto de trabajar con valores simples.

Los resultados de esta investigación y del índice presentado, permitirán establecer diálogos con las administraciones municipales y otros agentes urbanos, con el fin de plantear la necesidad de pensar y proyectar un diseño urbano sobre las calles de la ciudad pensando en las condiciones y necesidades de los peatones. Con este fin se empleó para el análisis espacial, datos que son accesibles y que comúnmente no se han utilizado para medir su influencia en la condición de caminar.

El artículo se estructura en seis secciones. En la primera se estudia, soportado en autores de referencia, que, para mejorar la caminata en la ciudad, no basta los indicadores físico espaciales, sino que deben tenerse en cuenta variables que permitan estudiar la experiencia del caminar, ya que son éstas las que generan una conexión con el lugar y estimulan el seguir repitiendo el tránsito por determinado lugar. En la segunda se contextualiza el caso de estudio que se emplea para el análisis, para pasar en la tercera sección a explicar la metodología empleada para realizar el índice. En la cuarta parte se muestran los resultados del índice aplicados al caso de estudio, el cual se confronta en la discusión y se concluye demostrando como las variables empleadas permiten visualizar otras acciones que deben tenerse en cuenta para diseñar ciudades caminables.

\section{MEJORAR LAS EXPERIENCIAS PARA LA CIUDAD CAMINABLE}

Durante el desarrollo de la investigación, el análisis se orientó a determinar los factores que favorecen caminar en un lugar. La revisión teórica determino un alto número de artículos que definían la importancia de caminar en la ciudad, y la necesidad de recuperar este espacio para el peatón. Si bien es común el reconocimiento del vehículo como el principal factor modificador del espacio urbano y con esto, de las relaciones humanas, parte de los estudios se centran en revisar las condiciones para caminar en relación a las dimensiones de los espacios y los conflictos que tiene el peatón con su principal rival, el vehículo.

La revisión de la literatura permitió entender que para hacer la ciudad caminable es importante considerar dos aspectos: la calidad espacial y la experiencia que se tiene en la calle para caminar (Ewing \& Clemente, 2013; Southworth, 2005; Leslie, et al.,2007; Gehl, 2014). Si además de la necesidad de desplazamiento que se realiza, este es ameno, esto hará que se incentive caminar una y otra vez por el mismo lugar (Gehl, 2014; Matos,2008).
La experiencia de la caminata se da por la estrecha relación entre esta acción, los sentidos, y el lugar donde ocurre. Matos (2008) menciona que, caminar es una práctica incorporada en la vida cotidiana que, aunque se realiza de forma natural, esta acción se va adaptando inconscientemente a las condiciones del contexto y las tareas que realiza. Con todas estas percepciones que recibe el cuerpo se va creando una noción del lugar en el inconsciente y se crean vínculos positivos y negativos con los sitios en los que se ha estado, haciendo que un lugar sea recordado por medio de las situaciones que allí se hayan vivido.

Los factores del diseño urbano que más influyen en la calidad espacial y en la escena urbana son, según (Ewing \& Clemente, 2013) la imagen, las condiciones del cerramiento de las fachadas, la escala humana, la transparencia y la complejidad. Sin embargo, estas no tienen el mismo grado de influencia. En el estudio que se aplicó para 526 manzanas de la ciudad de Nueva York, (Ewing et al., 2016) y luego de operacionalizar estas cinco cualidades en 20 características del paisaje urbano, concluyen que las variables que mayor inciden sobre la actividad peatonal, es decir, atraen a los peatones, son el mobiliario urbano, incluyendo su buena disposición y señalización; el porcentaje de usos activos, y la transparencia en el primer piso de la edificación. Leslie et al. (2007) y Southworth (2005), coinciden en trabajar con las variables de densidad y diversidad de usos y la conectividad. Southworth (2005), agrega en su trabajo la seguridad, la calidad física del trayecto, y la calidad de atracción que pueda tener el recorrido.

Las variables estudiadas en los autores de referencia (Southworth, 2005; Leslie, et al.,2007; Ewing \& Clemente, 2013; Gehl, 2014), permitieron validar las que se usarían para la investigación a partir de los recursos que disponíamos. Si bien no son exactamente las mismas por el acceso a la información, la investigación se centró en aquellas que permitieran evaluar factores similares como mejorar o alterar la experiencia de caminar, que en revisar las dimensiones y cualidades físico-espaciales que requerían mayor grado de precisión en la información y de la cual no se tenían datos.

Para optimizar los pocos datos disponibles, se definió, que para evaluar la experiencia de caminar existen factores positivos que incentivan la acción y factores negativos que desestimulan efectuarla. Fue así como se definieron la diversidad de usos y la densidad de árboles en los índices positivos y la pendiente de la vía (valoración del esfuerzo), la longitud del frente de manzana (menor accesibilidad) y el número de rutas de transporte público como índices negativos, considerando que el último indicador corresponde a buses con diésel y que no están articulados al sistema de transporte masivo de la ciudad.

Al realizar la correlación de las variables con el estudio de referencia, se tiene que la diversidad de usos aplica para la complejidad y atracción, la vegetación para la imagen, el frente de manzana da condiciones del cerramiento y conectividad, el esfuerzo para la escala humana, y la contaminación, aunque no es un elemento de diseño, condiciona la experiencia. 


\section{LA EXPERIENCIA DE CAMINAR EN MEDELLÍN. CASO DE ESTUDIO: CALLE SAN JUAN}

En el Plan de Ordenamiento Territorial vigente desde el 2014 para Medellín (principal instrumento de planificación de la ciudad), se invirtió la pirámide de movilidad, poniendo al peatón en la cima. Se determinó además, crear un plan de movilidad que permitiera mejorar esta situación en la ciudad. Sin embargo, las condiciones para que esto suceda están todavía lejos de ser reales. Por las circunstancias espaciales actuales, es conveniente orientar a la administración municipal en las variables que se deben en cuenta para mejorar las condiciones para caminar, y evitar se siga cayendo en el simplismo de la mejora material de andenes, donde no se está contemplando mínimamente su ampliación.

En el Plan de Movilidad Segura 2014-2020, se contempla el peatón cómo un "componente del tránsito" y las evaluaciones se realizaron en comparación al tránsito vehicular, pero en ningún momento se analiza la movilidad segura peatonal pensando en las personas, en los elementos que se requieren para mejorar el espacio para caminar y con esto, garantizar la seguridad del peatón y su bienestar. En el diagnóstico del mismo plan se encuentra citado: "La ciudad de Medellín cuenta en la actualidad con $114.876 \mathrm{~m} 2$ de zona peatonal, de los cuales el $75 \%$ se encuentra en buenas condiciones para el tránsito y comodidad de los peatones". (Alcaldía de Medellín, s.f.en Secretaria de movilidad de Medellin, 2014, p.99). permitiría tener una base de análisis para la toma de decisiones e incluso, adicionar otras variables según la información que se pueda obtener.

El estudio se realizó en la calle San Juan de la ciudad de Medellín. Se tomó esta calle por sus condiciones de localización, de movilidad, de comunicación, históricas y sociales. La calle San Juan es uno de los estructurantes principales de conexión transversal de la ciudad de Medellín (ver Figura 1)

Se encuentra en el centro de un valle largo y estrecho, por lo que es una de las calles de mayor extensión en kilómetros de la ciudad (ver Figura 1). Fue una de las primeras calles que atravesaron el río Medellín y establecieron la conexión entre el oriente y occidente de la ciudad. Fue históricamente una de las rutas principales del tranvía, y con su conexión y trazado en el sector occidental, constituyó el inicio del trazado urbano para este sector de la ciudad (CorreaRestrepo, 2002). Ha sido una importante vía de comunicación y conexión, integrando el sector de la América con el mercado de Guayaquil, como un importante centro de intercambio económico y hoy con el centro Administrativo municipal y regional. A partir de los años sesenta conecta también en sus cercanías con el Estadio municipal.

Según la encuesta origen - destino realizada por el Área Metropolitana del Valle de Aburrá (AMVA, 2017), de los viajes realizados entre las comunas de influencia de la calle, el $38 \%$ se realiza a pie y el $77 \%$ de estos viajes tiene un recorrido de menos de $2 \mathrm{Km}$. De la totalidad de estos viajes, el $9 \%$ se efectúan en transporte público, el $30 \%$ en auto y el $39 \%$ en bicicleta.

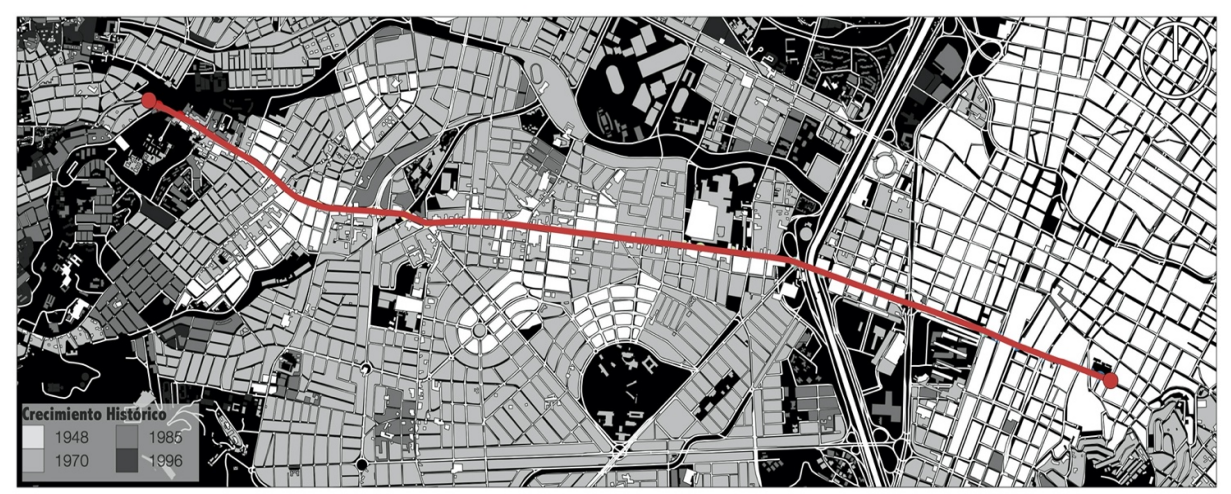

Figura 1. Crecimiento histórico del área urbana en el eje de la calle San Juan. Años 1948, 1970, 1985, 1996. Fuente: Estudio de la Forma y Crecimiento Urbano del Valle de Aburrá, (AMVA, 2007)

Dentro de este plan no queda claro que está definido dentro de estas zonas peatonales, pues los datos expuestos no corresponden a la realidad vivida en los andenes de la ciudad. El plan contempla el programa "En Medellín todos somos peatones" pero este está orientado a educar en los comportamientos, hábitos y conductas seguras de los usuarios de las vías, pero en ningún momento a mejorar las condiciones para que realmente caminar, como tipo de movilidad, sea más agradable y seguro. A falta de estudios más detallados sobre el tránsito peatonal en la ciudad, la construcción del índice
Es, por tanto, una calle de comunicación y conectividad es un importante eje de transporte público (aún no conectado al Sistema Integrado de Transporte del Valle de AburráSITVA-) pero teniendo estas características, no ha sido una calle donde se vea un gran número de peatones. Por estas razones se consideró como una calle óptima para efectuar los estudios sobre caminabilidad que nos planteamos y valorar los índices escogidos. 


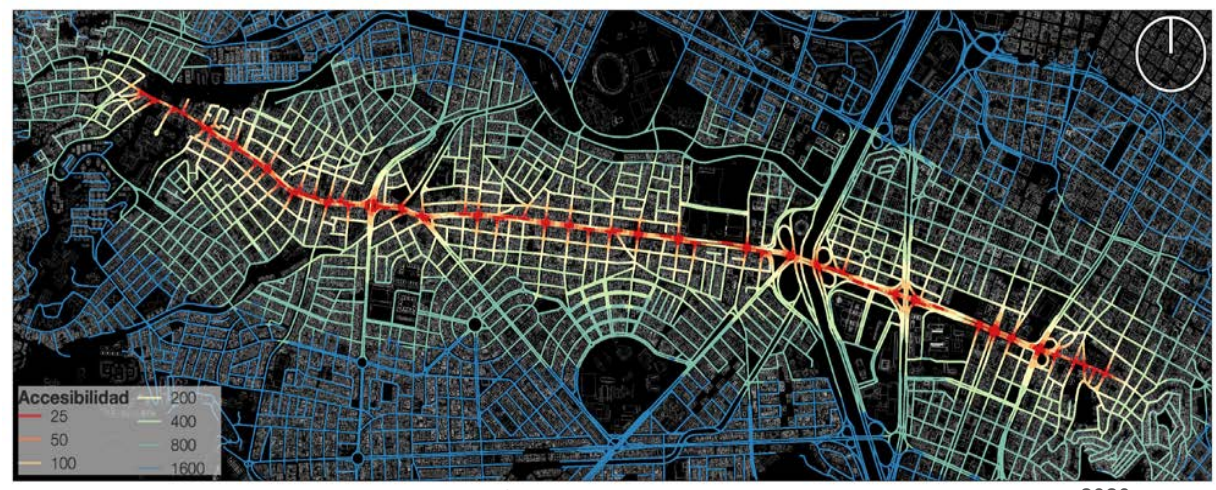

Figura 2. Accesibilidad en metros a la calle San Juan (Medellín) a través de la red víal. Elaboración Propia, 2020

\section{CONSTRUCCIÓN DEL ÍNDICE DE CAMINABILIDAD - METODOLOGÍA}

La definición conceptual presentada soporta un modelo espacial que incluye los valores urbanos necesarios para consolidar una ciudad caminable. Cada valor tiene una representación espacial especifica (punto, línea o polígono) y una distribución y concentración determinada a lo largo del corredor de la calle San Juan. Sin embargo, para la definición del modelo y su posterior validación, fue necesario entender la experiencia del caminar a través de tres métodos diferentes: el primero, que se desarrolla en este articulo, nos permite evaluar las áreas de influencia y los factores de atracción que tiene la calle a partir de información cartográfica referenciada; el segundo, nos ayuda a percibir los movimientos de las personas, cambios y obstáculos al caminar, a partir de aerofotografías y aerovuelos con drones; y por último, en la escala del peatón, uno que nos permitiera visualizar y medir la experiencia de caminar a partir de fichas de campo desarrolladas en el marco de la investigación, para lo cual se contó con apoyo de estudiantes. Estos dos últimos permiten validar y complementar el modelo espacial en una fase posterior de la investigación.

La calle San Juan permite diversas interacciones entre la zona occidental y la centralidad metropolitana del Valle de Aburrá, para el ejercicio, nos interesaba definir los limites de las posibles interacciones realizadas a pie. En relación con eso, la Encuesta Origen-Destino del Área Metropolitana del Valle de Aburrá, determino que el $99 \%$ de los viajes a pie se realizan en la primera etapa del trayecto y representan el $27 \%$ de los viajes totales realizados en los 10 municipios y, de esos viajes, el $34 \%$ recorren una distancia de entre 0-300 m. Esto ultimo, en correspondencia con la distancia aproximada que recorre una persona en 5 minutos, $375 \mathrm{~m}-425 \mathrm{~m}$, que como señala Gehl (Ciudades para la gente, 2014, p. 118), corresponde a la distancia "aceptable" para caminar en la ciudad (ver Figura 2).

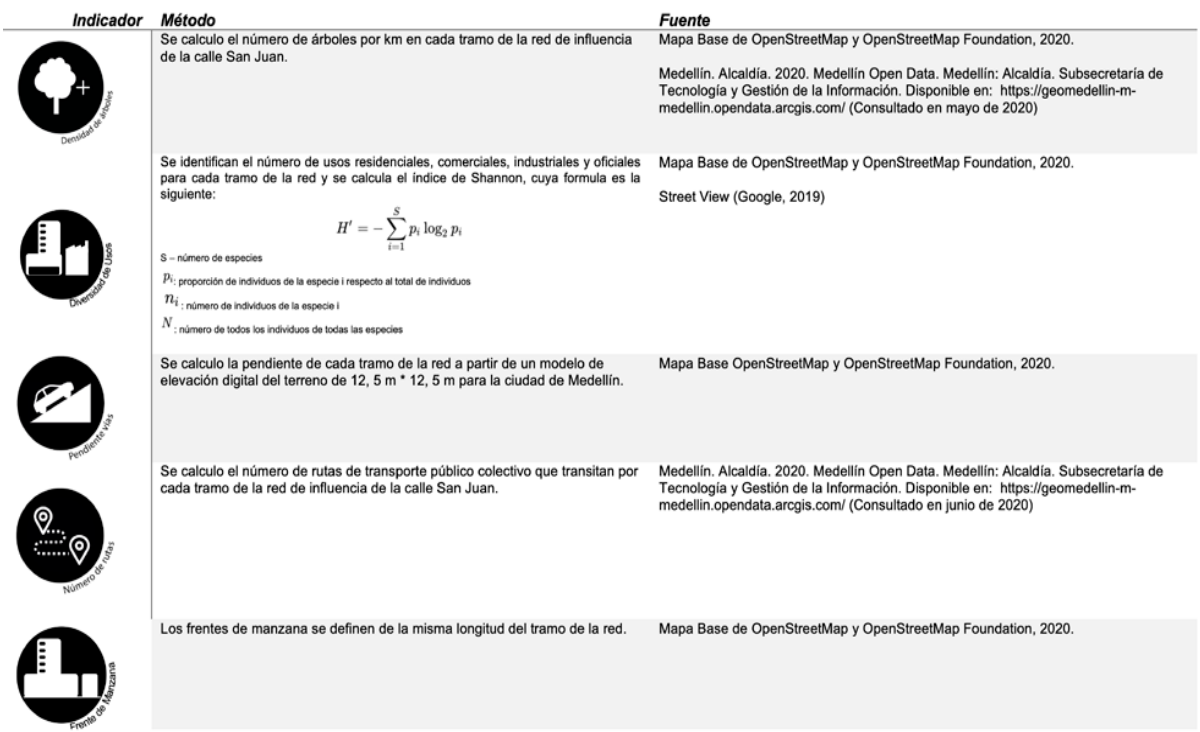

Figura 3. Indicadores Calculados. Elaboración propia 2020. 
El índice espacial multivariado, como síntesis del modelo propuesto, se construyo con los datos cartográficos a disposición, organizando la información en una matriz (Buzai \& Baxendale, 2006), donde las filas correspondian a las líneas de la red de influencia del corredor de la calle San Juan, y las columnas, a los indicadores o valores espaciales seleccionados a partir de la definición conceptual presentada. Para la selección de las variables de análisis se definieron, además, criterios de acceso, periodicidad en la actualización y escalabilidad de la información, con el fin de replicar el análisis en otras zonas urbanas con asimetrías en la gestión de datos espaciales.

El modelo espacial se elabora usando el software Arcgis 10.5 (Esri) e información cartográfica pública de diversas bases de datos (ver Figura 3)

La delimitación del área de estudio se realiza con un análisis de proximidad desde la Av San Juan a través de la red víal vehicular existente (no se dispone de informacion geografíca con el trazado peatonal), en donde, los tramos de la red en el área de influencia se definieron como unidad de análisis, y los indicadores anteriormente descritos, se calcularon a través de la herramienta SIG para cada línea que integra la red víal en el área de estudio.
A partir de los indicadores anteriores y luego de normalizar los valores para cada uno de los datos, se calculan los dos componentes del índice de caminabilidad; los positivos, que incluyen la densidad de arboles por $\mathrm{km}$ y la diversidad de usos; y los negativos, compuesto por la pendiente, el número de rutas y el frente de manzana. Cada uno de estos factores se elabora sin ponderar los indicadores internos, es decir, tanto en los positivos como en los negativos, las variables tienen el mismo peso en el calculo del factor, y el resultado se puede leer a continuación (ver Figuras 4 y 5 ):

Los factores positivos favorecen la caminabilidad y los factores negativos la restringen. Como se evidencia en las Figuras 3 y 4 , los tonos rojos en ambos casos representan las condiciones críticas y los azules, las propicias en la red de influencia de la calle San Juan.

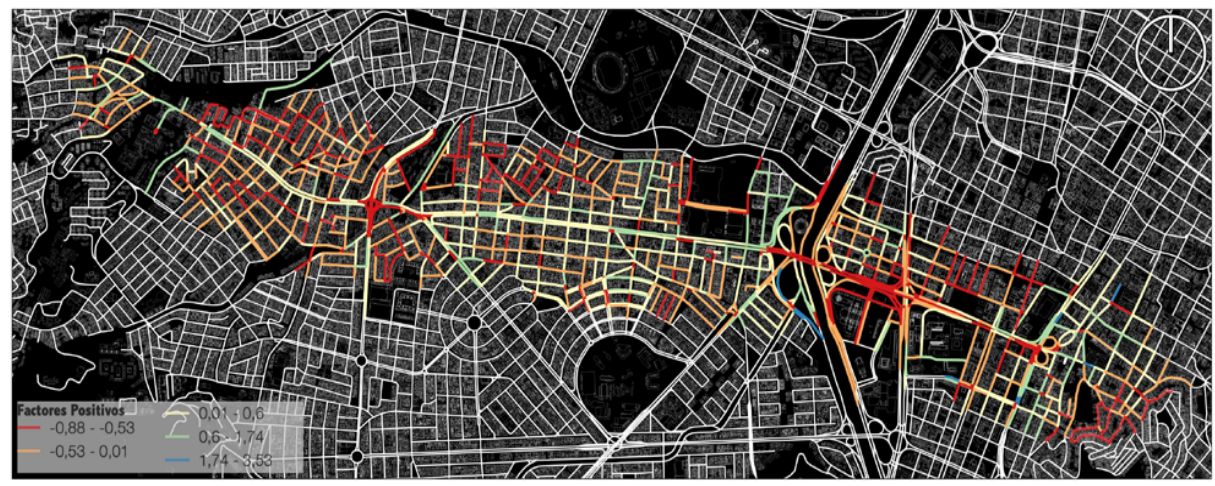

Figura 4. Factores positivos que favorecen la caminabilidad, Elaboración Propia, 2020

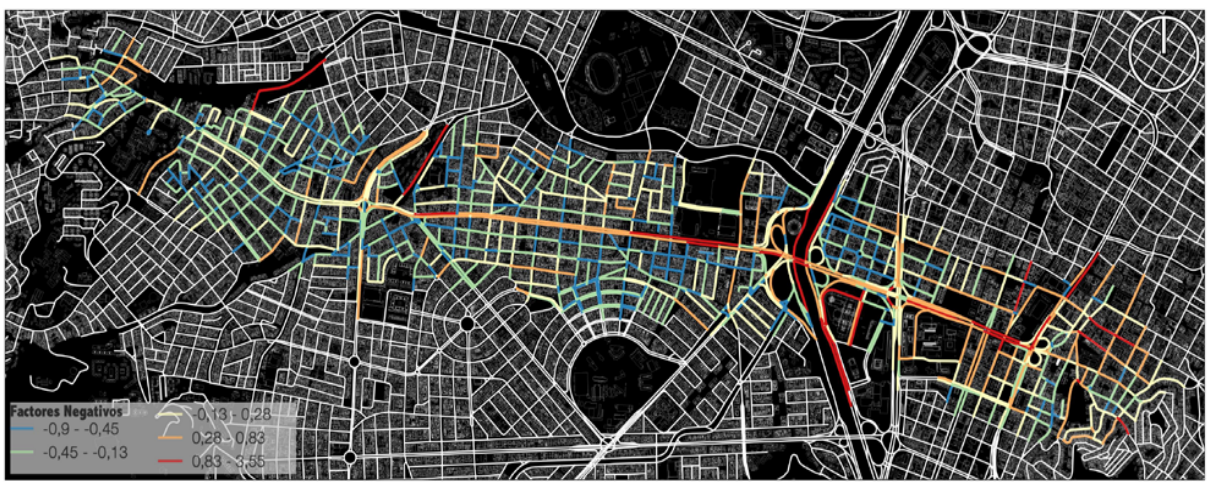

Figura 5. Factores negativos que restringen la caminabilidad, Elaboración Propia, 2020. 


\section{RESULTADOS}

Las variables que se determinaron para el análisis recibieron el mismo valor de ponderación. Primero porque al ser pocas, eran representativas para valorar las cualidades escénicas que eran relevantes para la evaluación de la calidad del diseño urbano como se expuso en el soporte conceptual. Segundo, porque era necesario unificar el peso de las variables, de manera que permitiera determinar cuáles inciden más sobre el factor de la caminata y su experiencia a partir de su relación en el contexto sobre el que se realiza el análisis. El resultado del índice se puede evidenciar en la Figura 6.
Los datos se representan en 5 rangos clasificados con el método de quiebres naturales (Jenks) y evidencian, valores muy bajos sobre la calle San Juan en la zona oriental de la red entre la Av. Regional y la Av. Oriental; valores medios y altos en la parte media de la calle con variaciones entre la zona norte y sur de la red; y valores medio bajos en la zona occidental del área de estudio. Las diferencias son consecuentes con las condiciones heterogéneas de los factores en el área de estudio, y con la concentración de valores positivos en la zona centro sur de la red con condiciones favorables tanto en pendientes como en frentes de manzana por la estructura morfológica existente.

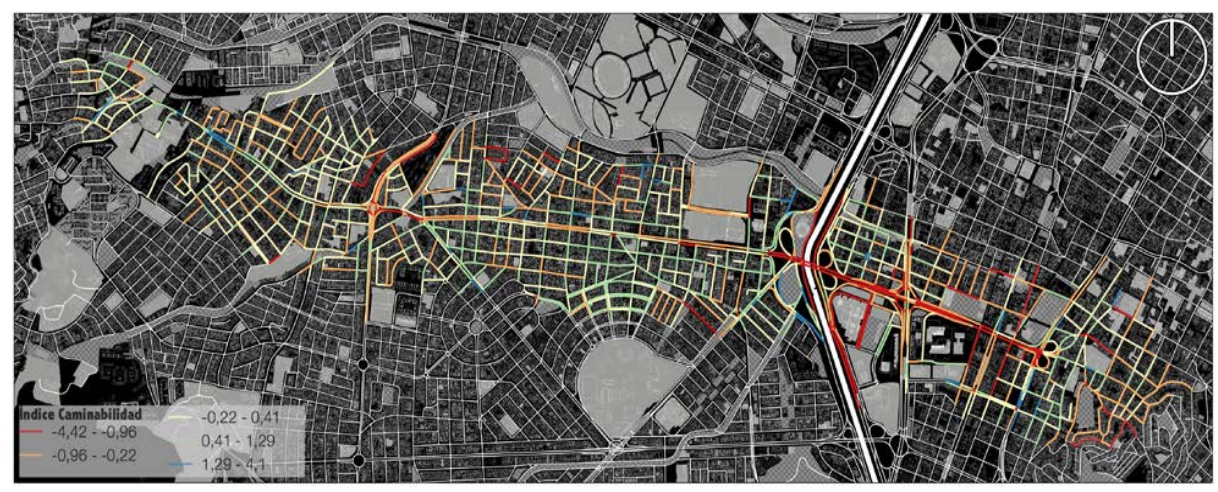

Figura 6. Índice de caminabilidad para la red de influencia de la calle San Juan, Medellín. Elaboración Propia, 2020.

Imagen de referencia Indice de Caminabilidad
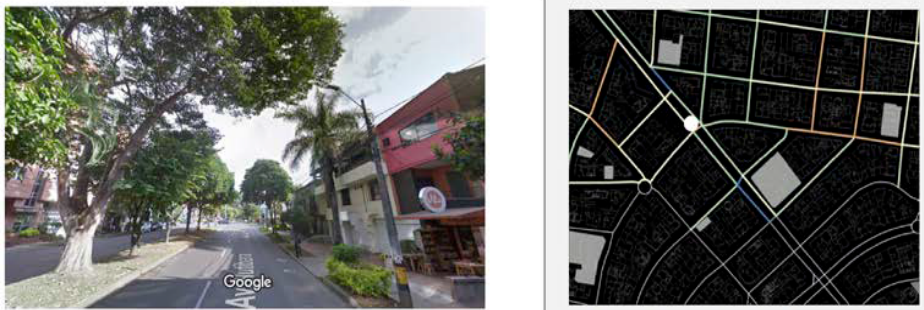

Indicador

Valor

Densidad Arboles $\quad 1,18$

Diversidad Usos $\quad 0,51$

Pendiente $-0,38$

\#-Rutas

$-0,76$

Frente Manzana

0,28

1,20

Indice
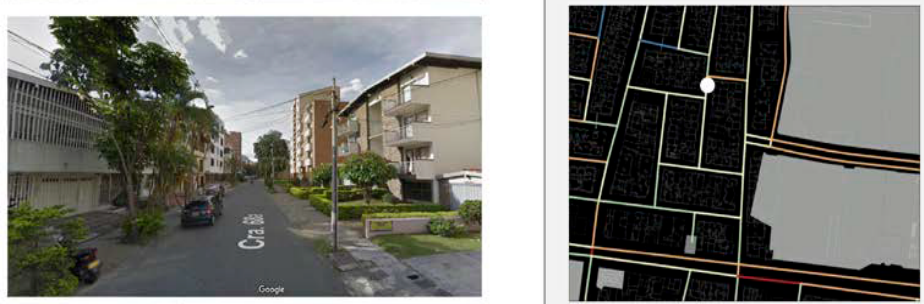

Densidad Arboles

$-0,58$

Diversidad Usos

0,46

Pendiente

$-0,54$

\#-Rutas

$-0,76$

Frente Manzana

Indice

0,29
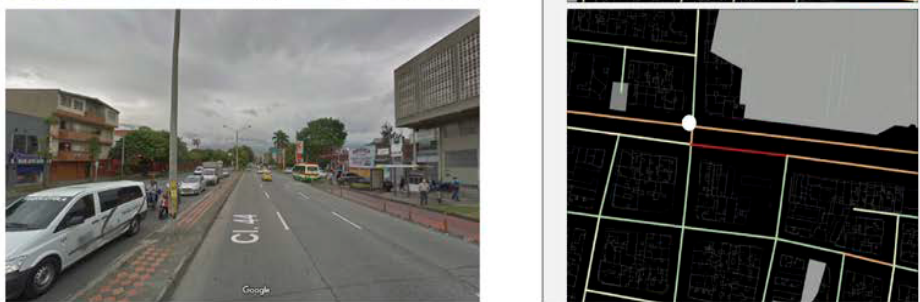

Densidad Arboles

$-0,89$

Diversidad Usos

Pendiente

$-0,22$

\#-Rutas

0,66

Frente Manzana

0,25

Indice

Figura 7. Índice de caminabilidad obtenido en tres tramos de la red de análisis. Elaboración Propia, 2020. 
Para una mayor eficacia en el diseño urbano, detectar cual es el grado de incidencia de los factores en determinados tramos de la red, permite tener estrategias diferenciadoras en el diseño de la calle y con esto, lograr la mejora de la calidad escénica de la misma. Para los corredores peatonales definidos en el Plan de Ordenamiento de Medellín (Acuerdo 48, 2014) localizados en la red estudiada, por ejemplo, el índice permitiría ajustar los factores que favorecen la caminabilidad.

\section{DISCUSIÓN}

El índice de caminata presentado es un trabajo exploratorio que permite poner en términos espaciales diversas caracteristicas que permiten evaluar las condiciones para caminar que puede tener un eje de la ciudad y que son analizables desde la escala del diseño urbano. Este índice permite reconocer cuales son las áreas críticas que inciden sobre la caminata. Las características de la mayor parte de las calles de la ciudad de Medellín en la actualidad, no ofrece las condiciones efectivas para que las personas elijan caminar como primera opción de desplazamiento y el índice valida los criterios que afectan estas decisiones.

Las variables empleadas en el índice coinciden con los trabajados por Leslie et al. (2007), Southworth (2005) y Ewing \& Clemente (2013) en los cuales se hace énfasis en el tema de densidad y diversidad de usos, como factores claves para incrementar la atracción del trayecto. Asimismo, estos estudios señalan la conectividad la cual es evaluada a partir de la frecuencia en las intersecciones de las calles, que para el caso presentado en esta publicación se analiza a través de la longitud del frente de manzana. Por los datos a los que se tenía acceso y la escala trabajada, el factor de calidad espacial (Southworth, 2005; Ewing \& Clemente, 2013; Gehl, 2014) se evalúa sólo por la condición de densidad de árboles, y que se puede ampliar en otra etapa del análisis integrando factores más específicos como las dimensiones del andén y la transparencia de la fachada, que se señalan en estos mismos artículos y que es de las variables más coincidentes dentro de la literatura que relaciona las condiciones para caminar. El factor de la integración a las redes de transporte que menciona Southworth (2005), para este índice se califica como un factor negativo puesto que como se mencionó anteriormente, la red de transporte que pasa a lo largo del eje de la calle San Juan, no se encuentra articulado al resto de la red de transporte de la ciudad y por funcionar por diésel, es un factor altamente contaminante, que incide negativamente sobre la elección de caminar.

Este índice es el resultado de una primera etapa de investigación, pero no constituye en su totalidad una evaluación de la experiencia de caminar. Aunque las variables utilizadas en el índice buscaban aproximarse a los valores que más se acercaran a poder medir la experiencia como lo mencionan en sus estudios Ewing \& Clemente (2013) y Southworth (2005), para tener un resultado más completo, es necesario poder evaluar la experiencia individual y colectiva del caminar mediante indicadores que se puedan medir desde el trabajo de campo, como lo evidencian los estudios citados.
A futuro, dado que este índice es exploratorio, se puede integrar variables más específicas y que involucre otras escalas de trabajo más detalladas como la referencia de los estudios de Gehl (2014), Ewing \& Clemente (2013) y Ewing et al. (2016). El índice puede potenciarse con los resultados que se puedan obtener a través de fichas de trabajo en campo y que evalúen la experiencia de caminar, y además, complementarse, a partir de otros datos a los que se pueda acceder con un apoyo directo desde las entidades territoriales.

Los trabajos de Frank, et al. (2010) y Leslie, et al.(2007), sugieren además, el encuentro con otras disciplinas como la medicina y la salud pública, que además de aportar otras variables del análisis, pueden sugerir otras interpretaciones para la aplicación del índice, entre las cuales se exponen la diferenciación por grupos etarios e integración de otras herramientas como encuestas que asocian la caminata con la salud.

Los índices establecidos mediante Sistemas de Información Geográfica, requieren a su vez validarse en sitio, como lo demuestran en su estudio Leslie, et al. (2007). Para la realización del índice presentado en este trabajo, el sitio, es decir, el contexto que se definió como caso de estudio, fue la base para la construcción del mismo. A partir de las experiencias, análisis y conocimientos previos que se tenían sobre el lugar, se seleccionaron las variables que con mayor claridad podían demostrar las afectaciones sobre la caminata y que coincidieran con la validación de las variables identificadas en los estudios sobre el tema. Sin embargo, como ya se ha mencionado, poder integrar otros indicadores de análisis desde el trabajo de campo, permitirían a su vez, dar una nueva validación al índice. Otro punto que está pendiente de realizar es la aplicación del índice en otras calles, zonas de la ciudad, y por supuesto otras ciudades, como el caso de Montería que se incluye en el estudio.

En un contexto de información restringido y costoso de levantar, el índice no integra todas las variables que se enumeran en la literatura. Este estudio, sin embargo, ha permitido identificar cómo variables que se asocian a la experiencia que puede tener una persona en la calle que camina y que no han sido frecuentemente analizadas con el enfoque sobre la calidad escénica, deben tenerse en cuenta para el análisis y el diseño urbano, y servir de complemento, a los análisis tradicionales de calidad espacial.

\section{CONCLUSIONES}

Esta investigación busca con la creación del índice, poder evaluar experiencias similares a las creadas por Ewing \& Clemente (2013) y Ewing et al. (2016), pero al contrario de estos análisis más robustos y complejos, se pretende usar datos más accesibles que nos permita implementar el modelo a pesar de las asimetrías de la información o las condiciones heterogéneas de distintas áreas urbanas. El análisis realizado nos permite concluir y aclarar que no se puede valorar el índice de caminata únicamente con las variables acá utilizadas, valorar esta experiencia incluye integrar otros componentes y escalas de análisis. Sin embargo, la aplicación de este índice permite identificar particularidades y tomar decisiones a las administraciones públicas sobre qué elementos priorizar, cuáles tramos 
integrar y que componentes mejorar en el diseño urbano de las calles. Este índice, en síntesis, complementado con variables tales como concentración de personas; ancho y estados de los andes; ancho de la calzada, y continuidad espacial, mejora la identificación de los tramos de intervención; facilita la priorización y favorece la implementación de las estrategias proyectuales.

El modelo de análisis presentado busca validar, a partir de los datos abiertos accesibles, indicadores que no se habían utilizado en la ciudad y que permiten verificar la calidad de la escena urbana y con esto, como favorecen o restringen la posibilidad de caminar por un lugar. Los resultados mostrados con el índice permiten identificar situaciones diferenciadas en distintos trayectos de la calle analizada y sus áreas de influencia, lo que posibilita ser más precisos en el tipo de estrategias que se requiere realizar y con esto dar pautas para el diseño urbano, como también la priorización de segmentos o lugares para intervenir.

El análisis espacial a través del SIG permitió generar un modelo de análisis para comparar un entorno heterogéneo como la calle San Juan, además de ser un modelo dinámico en el que se pueden actualizar los datos y ajustar los criterios de intervención. Estas modelaciones arrojan resultados de fácil interpretación y que permiten tomar decisiones de manera eficaz. La escala del análisis empleada permitió llegar a resultados que inciden sobre el diseño urbano, no sólo a la escala de la planificación territorial. En el contexto colombiano, la calidad en el diseño del espacio urbano aún está poco valorada y aún las políticas de movilidad se realizan sobre la infraestructura, pero no sobre la mejora del diseño espacial de las calles, que es, al final, el espacio que habitamos todos los días. Mejorar las condiciones espaciales de las calles puede incrementar la caminata, pero para esto es importante la valoración de los factores que incentivan este modo de desplazamiento, que al final repercute en una mejora de la calidad urbana para todos los habitantes.

\section{REFERENCIAS}

AMVA, Area Metropolitana del Valle de Aburrá. (2017). Encuesta origen destino.

AMVA.

https://www.metropol.gov.co/observatorio/Paginas/encuest aorigendestino.aspx

Buzai, G., \& Baxendale, C. (2006). Análisis por clasificación multivariada de unidades espaciales. In Análisis socioespacial con Sistemas de Información Geográfica (p. 397). Ed. Lugar.

CorreaRestrepo, J. S. (2002). Urbanismo y transporte: el tranvía de Medellín (1919-1950). Documentos de Trabajo UEC. https://ideas.repec.org/p/col/000139/003740.html

Ewing, R., \& Clemente, O. (2013). Measuring Urban Design: Metrics for Livable Places (IslandPress (ed.)).

Ewing, R., Hajrasouliha, A., Neckerman, K. M., Purciel-Hill, M., \& Greene, W. (2016). Streetscape Features Related to Pedestrian Activity. Journal of Planning Education and Research, 36(1), 5-15. https://doi.org/10.1177/0739456X15591585

Frank, L. D., Sallis, J. F., Saelens, B. E., Leary, L., Cain, L., Conway, T. L., \& Hess, P. M. (2010). The development of a walkability index: Application to the neighborhood quality of life study. British Journal of Sports Medicine, 44(13), 924933. https://doi.org/10.1136/bjsm.2009.058701

Gehl, J. (2014). Ciudades para la gente. http://www.edicionesinfinito.com

Leslie, E., Coffee, N., Frank, L., Owen, N., Bauman, A., \& Hugo, G. (2007). Walkability of local communities: Using geographic information systems to objectively assess relevant environmental attributes. Health and Place, 13(1), 111-122. https://doi.org/10.1016/j.healthplace.2005.11.001

Matos Wunderlich, F. (2008). Walking and Rhythmicity: Sensing Urban Space. Journal of Urban Design, 13(1), 125-139. https://doi.org/10.1080/13574800701803472

Secretaria de movilidad de Medellin. (2014). Plan de Movilidad Segura de Medellín 2014 - 2020 - Secretaría de Movilidad de Medellín. Alcaldía de Medellín. https://www.medellin.gov.co/movilidad/secretaria-demovilidad/plan-de-movilidad-segura-de-medellin-20142020

Southworth, M. (2005). Designing the walkable city. Journal of Urban Planning and Development, 131(4), 246-257. https://doi.org/10.1061/(ASCE)07339488(2005)131:4(246)

Universidad Pontificia Bolivariana; Área Metropolitana del Valle de Aburrá. (2007). Estudio de la forma y el crecimiento urbano de la región metropolitana: Fase 1: mapificación del crecimiento urbano del Valle de Aburrá. Medellín. 